\title{
New generalized reverse Minkowski and related integral inequalities involving generalized fractional conformable integrals
}

\author{
Saima Rashid', Ahmet Ocak Akdemir², Kottakkaran Sooppy Nisar ${ }^{3}$, Thabet Abdeljawad ${ }^{4,5,6^{*}}$ (D) and \\ Gauhar Rahman
}

*Correspondence:
tabdeljawad@psu.edu.sa
${ }^{4}$ Department of Mathematics and
General Sciences, Prince Sultan
University, 66833 Riyadh, Saudi
Arabia
${ }^{5}$ Department of Medical Research,
China Medical University, 40402
Taichung, Taiwan
Full list of author information is
available at the end of the article

\section{Springer}

\begin{abstract}
This paper gives some novel generalizations by considering the generalized conformable fractional integrals operator for reverse Minkowski type and reverse Hölder type inequalities. Furthermore, novel consequences connected with this inequality, together with statements and confirmation of various variants for the advocated generalized conformable fractional integral operator, are elaborated. Moreover, our derived results are provided to show comparisons of convergence between old and modified operators towards a function under different parameters and conditions. The numerical approximations of our consequence have several utilities in applied sciences and fractional integro-differential equations.
\end{abstract}

MSC: 26D15; 26D20; 26D07

Keywords: Integral inequality; Minkowski inequality; Reverse Minkowski inequality; Conformable integrals; Generalized conformable fractional integral operators

\section{Introduction}

Fractional calculus, generally referred to as the calculus of non-integer order, was a trademark outgrowth of traditional definitions of calculus integral and derivative. The concept of fractional calculus has provoked a host of researchers and was comprehensively studied in the literature for the last few decades. A continuous effort has been made on an enormous scale and everybody has been stimulated by its different aspects. In the present century, the exceptional idea has been described by several mathematicians with a slightly distinct technique in different time scales; see, for instance, the Liouville, Riemann, Grunwald, Letnikov, Hadamard, Weyl, Riesz, Marchaud, Kober and Caputo fractional integrals (see [1-11]). Most of these researchers first of all added fractional integrals, on the concept of which the associated fractional derivative and other associated results had been produced. Recently, Khalil et al. [2] and Abdeljawad [1] introduced fractional operators known as fractional conformable derivatives and integrals. Jarad et al. [12] established the fractional conformable integral operators. Meanwhile in [13], Anderson and Ulness introduced the concept of local derivatives for upgrading the concept of the fractional

(c) The Author(s) 2020. This article is licensed under a Creative Commons Attribution 4.0 International License, which permits use sharing, adaptation, distribution and reproduction in any medium or format, as long as you give appropriate credit to the original author(s) and the source, provide a link to the Creative Commons licence, and indicate if changes were made. The images or other third party material in this article are included in the article's Creative Commons licence, unless indicated otherwise in a credit line to the material. If material is not included in the article's Creative Commons licence and your intended use is not permitted by statutory regulation or exceeds the permitted use, you will need to obtain permission directly from the copyright holder. To view a copy of this licence, visit http://creativecommons.org/licenses/by/4.0/. 
conformable derivative. The exponential and Mittag-Leffler functions are used as kernels by several researchers for developing new fractional techniques. In [14], Khan et al. established a new class of generalized conformable fractional integral operators. Such generalizations encourage future studies requiring extra thoughts to merge the fractional operators and achieve the variants regarding such fractional operators.

Conformable derivatives are nonlocal fractional derivatives. They can be called fractional since we can take derivatives up to arbitrary order. However, since in the community of fractional calculus, nonlocal fractional derivatives only are to be called fractional, we prefer to replace conformable fractional by conformable (as a type of local fractional). Conformable derivatives and other types of local fractional derivatives or modified conformable derivatives in [13] can gain in importance by the ability to use them to generate more generalized nonlocal fractional derivatives with singular kernels (see [15-22]).

Integral inequalities have potential application in several areas of science: technology, mathematics, chemistry, plasma physics, among others; especially we point out initial value problems, the stability of linear transformation, integral differential equations, and impulse equations [23-33]. Variants regarding fractional integral operators are of use in significant strategies amongst researchers and accumulate fertile functional applications in various areas of science; see [34-45]. On account of their potential results to be utilized for the presence of nontrivial and positive solutions of a distinct kind of fractional differential equations, our findings concerning fractional integrals are appreciable and essential.

An enormous heft of present literature comprises generalizations of several variants by fractional integral operators and their applications [46-52]. We state some of them, that is, the variants of Minkowski, Hardy, Opial, Hermite-Hadamard, Grüss, Lyenger, Wrtinger, Ostrowski, Čebyšev and Pólya-Szegö [53-59]. Such applications of fractional integral operators compelled us to show the generalization of the reverse Minkowski inequality $[43,44,53]$ involving generalized conformable fractional integrals operators.

The article is composed thus: in Sect. 2 we demonstrate the notations and primary definitions of our newly introduced operator generalized conformable fractional integrals. Also, we present the results concerning the reverse Minkowski inequality. In Sect. 3, we advocate essential consequences such as the reverse Minkowski inequality via the generalized conformable fractional integral operators. In Sect. 4, we show the associated variants using this fractional integral.

\section{Preliminaries}

This section is dedicated to some recognized definitions and results associated with the generalized conformable fractional integral operators and their generalization related to the generalized conformable fractional integral operators. Set et al. in [60] proved the Hermite-Hadamard, and reverse Minkowski inequalities for Riemann-Liouville fractional integrals. Additionally, Hardy's type and reverse Minkowski inequalities were supplied by Bougoffa in [38]. The subsequent consequences concerning the reverse Minkowski inequalities are of significance for the classical integrals. 
Theorem 2.1 ([60]) For $p \geq 1$ and let there be two positive functions $f_{1}$ and $f_{2}$ on $[0, \infty)$. If $0<\theta_{1} \leq \frac{f_{1}(\eta)}{f_{2}(\eta)} \leq \theta_{2}, y \in\left[r_{1}, r_{2}\right]$, then

$$
\left(\int_{r_{1}}^{r_{2}} f_{1}^{p}(y) d y\right)^{\frac{1}{p}}+\left(\int_{r_{1}}^{r_{2}} f_{2}^{p}(y) d y\right)^{\frac{1}{p}} \leq \frac{1+\theta_{2}\left(\theta_{1}+2\right)}{\left(\theta_{1}+1\right)\left(\theta_{2}+1\right)}\left(\int_{r_{1}}^{r_{2}}\left(f_{1}+f_{2}\right)^{p}(y) d y\right)^{\frac{1}{p}} .
$$

Theorem 2.2 ([60]) For $p \geq 1$ and let there be two positive functions $f_{1}$ and $f_{2}$ on $[0, \infty)$. If $0<\theta_{1} \leq \frac{f_{1}(\eta)}{f_{2}(\eta)} \leq \theta_{2}, y \in\left[r_{1}, r_{2}\right]$, then

$$
\begin{aligned}
& \left(\int_{r_{1}}^{r_{2}} f_{1}^{p}(y) d y\right)^{\frac{2}{p}}+\left(\int_{r_{1}}^{r_{2}} f_{2}^{p}(y) d y\right)^{\frac{2}{p}} \\
& \quad \geq\left(\frac{\left(1+\theta_{2}\right)\left(\theta_{1}+1\right)}{\theta_{2}}-2\right)\left(\int_{r_{1}}^{r_{2}} f_{1}^{p}(y) d y\right)^{\frac{1}{p}}\left(\int_{r_{1}}^{r_{2}} f_{2}^{p}(y) d y\right)^{\frac{1}{p}} .
\end{aligned}
$$

In [44], Dahmani used the Riemann-Liouville fractional integral operators to prove the subsequent reverse Minkowski inequalities.

Theorem 2.3 ([44]) Let $\varsigma>0$ and $p \geq 1$, and let there be two positive functions $f_{1}$ and $f_{2}$ on $[0, \infty)$ such that, for all $y>0, \mathcal{K}_{r_{1}^{+}}^{5} f_{1}^{p}(y)<\infty, \mathcal{K}_{r_{1}^{+}}^{5} f_{2}^{p}(y)<\infty$. If $0<\theta_{1} \leq \frac{f_{1}(\eta)}{f_{2}(\eta)} \leq \theta_{2}, \eta \in\left[r_{1}, y\right]$, then the following inequality holds:

$$
\left(\mathcal{K}_{r_{1}^{+}}^{\varsigma} f_{1}^{p}(y)\right)^{\frac{1}{p}}+\left(\mathcal{K}_{r_{1}^{+}}^{\varsigma} f_{2}^{p}(y)\right)^{\frac{1}{p}} \leq \frac{1+\theta_{2}\left(\theta_{1}+2\right)}{\left(\theta_{1}+1\right)\left(\theta_{2}+1\right)}\left(\mathcal{K}_{r_{1}^{+}}^{\varsigma}\left(f_{1}+f_{2}\right)^{p}(y)\right)^{\frac{1}{p}}
$$

Theorem 2.4 ([44]) Let $\varsigma>0$ and $p \geq 1$, and let there be two positive functions $f_{1}$ and $f_{2}$ on $[0, \infty)$ such that, for all $y>0, \mathcal{K}_{r_{1}^{+}}^{5} f_{1}^{p}(y)<\infty, \mathcal{K}_{r_{1}^{+}}^{5} f_{2}^{p}(y)<\infty$. If $0<\theta_{1} \leq \frac{f_{1}(\eta)}{f_{2}(\eta)} \leq \theta_{2}, \eta \in\left[r_{1}, y\right]$, then the following inequality holds:

$$
\left(\mathcal{K}_{r_{1}^{+}}^{\varsigma} f_{1}^{p}(y)\right)^{\frac{2}{p}}+\left(\mathcal{K}_{r_{1}^{+}}^{\varsigma} f_{2}^{p}(y)\right)^{\frac{2}{p}} \geq\left(\frac{\left(1+\theta_{2}\right)\left(\theta_{1}+1\right)}{\theta_{2}}-2\right)\left(\mathcal{K}_{r_{1}^{+}}^{\varsigma} f_{1}^{p}(y)\right)^{\frac{1}{p}}\left(\mathcal{K}_{r_{1}^{+}}^{\varsigma} f_{2}^{p}(y)\right)^{\frac{1}{p}}
$$

Recall the definition of the generalized conformable fractional integral which is mainly due to [14].

Definition 2.5 ([14]) Let $f$ be a conformable integrable function on the interval $\left[r_{1}, r_{2}\right] \subseteq$ $[0, \infty)$. The right-sided and left-sided generalized conformable fractional integrals ${ }_{\varrho}^{\tau} \mathcal{K}_{r_{1}^{+}}^{\varsigma}$ and ${ }_{\varrho}^{\tau} \mathcal{K}_{r_{2}^{-}}^{\zeta}$ of order $\varsigma>0$ are defined by

$$
{ }_{\varrho}^{\tau} \mathcal{K}_{r_{1}^{+}}^{\varsigma} f(y)=\frac{1}{\Gamma(\varsigma)} \int_{r_{1}}^{y}\left(\frac{y^{\tau+\varrho}-\eta^{\tau+\varrho}}{\tau+\varrho}\right)^{\varsigma-1} \frac{f(\eta)}{\eta^{1-\tau-\varrho}} d \eta, \quad y>r_{1},
$$

and

$$
{ }_{\varrho}^{\tau} \mathcal{K}_{r_{2}^{-}}^{\varsigma} f(y)=\frac{1}{\Gamma(\varsigma)} \int_{y}^{r_{2}}\left(\frac{\eta^{\tau+\varrho}-y^{\tau+\varrho}}{\tau+\varrho}\right)^{\varsigma-1} \frac{f(\eta)}{\eta^{1-\tau-\varrho}} d \eta, \quad y<r_{2}
$$

where $\varsigma \in \mathbb{C}, \mathfrak{R}(\varsigma)>0, \varrho \in(0,1], \tau \in \mathcal{R}$ with $\tau+\varrho \neq 0$, and $\Gamma$ is the well-known gamma function. 
Remark 2.6 In Eqs. (2.1) and (2.2):

(i) If $\tau=0$, then we attain the subsequent Riemann-Liouville type fractional conformable integral operators; see [12]:

$$
{ }_{\varrho} \mathcal{K}_{r_{1}^{+}}^{\varsigma} f(y)=\frac{1}{\Gamma(\varsigma)} \int_{r_{1}}^{y}\left(\frac{y^{\varrho}-\eta^{\varrho}}{\varrho}\right)^{\varsigma-1} \frac{f(\eta)}{\eta^{1-\varrho}} d \eta, \quad y>r_{1}
$$

and

$$
{ }_{\varrho} \mathcal{K}_{r_{2}}^{\zeta} f(y)=\frac{1}{\Gamma(\varsigma)} \int_{y}^{r_{2}}\left(\frac{\eta^{\varrho}-y^{\varrho}}{\varrho}\right)^{\varsigma-1} \frac{f(\eta)}{\eta^{1-\varrho}} d \eta, \quad y<r_{2}
$$

where $\varsigma \in \mathbb{C}, \mathfrak{R}(\varsigma)>0, \varrho \in(0,1]$.

(ii) If $\tau=0$ and $\varrho=1$, then we attain the subsequent Riemann-Liouville type fractional integral operators; see [10, 15]:

$$
\mathcal{K}_{r_{1}^{+}}^{\varsigma} f(y)=\frac{1}{\Gamma(\varsigma)} \int_{r_{1}}^{y}(y-\eta)^{\varsigma-1} f(\eta) d \eta, \quad y>r_{1}
$$

and

$$
\mathcal{K}_{r_{2}^{-}}^{\varsigma} f(y)=\frac{1}{\Gamma(\varsigma)} \int_{y}^{r_{2}}(\eta-y)^{\varsigma-1} f(\eta) d \eta, \quad y<r_{2}
$$

where $\varsigma \in \mathbb{C}, \mathfrak{R}(\varsigma)>0$.

\section{Reverse Minkowski inequalities via generalized conformable fractional integral operators}

This section comprises our principal involvement of establishing the proof of the reverse Minkowski inequalities via generalized conformable fractional integral operators defined in (2.1) and (2.2) and an associated theorem insinuated as the reverse Minkowski inequalities.

Theorem 3.1 For $\varsigma>0, \varrho \in(0,1], \tau \in \mathcal{R}$ and $\varrho+\tau \neq 0$ with $p \geq 1$ and let there be two positive functions $f_{1}, f_{2}$ on $[0, \infty)$ such that, for all $y>r_{1},{ }_{\varrho}^{\tau} \mathcal{K}_{r_{1}^{5}}^{5} f_{1}^{p}(y)<\infty$ and ${ }_{\varrho}^{y} \mathcal{K}_{r_{1}^{5}}^{5} f_{2}^{p}(y)<\infty$. If $0<\theta_{1} \leq \frac{f_{1}(\eta)}{f_{2}(\eta)} \leq \theta_{2}$ for $\theta_{1}, \theta_{2} \in \mathcal{R}^{+}$and for all $x \in\left[r_{1}, y\right]$, then

$$
\left({ }_{\varrho}^{y} \mathcal{K}_{r_{1}^{+}}^{\varsigma} f_{1}^{p}(y)\right)^{\frac{1}{p}}+\left({ }_{\varrho}^{y} \mathcal{K}_{r_{1}^{+}}^{\varsigma} f_{2}^{p}(y)\right)^{\frac{1}{p}} \leq \frac{1+\theta_{2}\left(\theta_{1}+2\right)}{\left(\theta_{1}+1\right)\left(\theta_{2}+1\right)}\left({ }_{\varrho}^{y} \mathcal{K}_{r_{1}^{+}}^{\varsigma}\left(f_{1}+f_{2}\right)^{p}(y)\right)^{\frac{1}{p}}
$$

Proof By the suppositions mentioned in Theorem 3.1, $\frac{f_{1}(\eta)}{f_{2}(\eta)} \leq \theta_{2}, r_{1} \leq \eta \leq y$, we have

$$
(M+1)^{p} f_{1}^{p}(\eta) \leq M^{p}\left(f_{1}(\eta)+f_{2}(\eta)\right)^{p} .
$$


If we multiply both sides of (3.2) with $\frac{1}{\Gamma(\varsigma) \varsigma^{1-\tau-\varrho}}\left(\frac{y^{\tau+\varrho}-\eta^{\tau+\varrho}}{\tau+\varrho}\right)^{\tau-1}$ and then integrate the subsequent inequality with respect to $\eta$ from $r_{1}$ to $y$, we obtain

$$
\begin{aligned}
& \frac{(M+1)^{p}}{\Gamma(\varsigma)} \int_{r_{1}}^{y}\left(\frac{y^{\tau+\varrho}-\eta^{\tau+\varrho}}{\tau+\varrho}\right)^{\tau-1} \frac{f_{1}^{p}(\eta)}{\varsigma^{1-\tau-\varrho}} d \eta \\
& \leq \frac{M^{p}}{\Gamma(\varsigma)} \int_{r_{1}}^{y}\left(\frac{y^{\tau+\varrho}-\eta^{\tau+\varrho}}{\tau+\varrho}\right)^{\tau-1} \frac{\left(f_{1}(\eta)+f_{2}(\eta)\right)^{p}}{\varsigma^{1-\tau-\varrho}} d \eta .
\end{aligned}
$$

Similarly,

$$
\left({ }_{\varrho}^{y} \mathcal{K}_{r_{1}^{+}}^{\varsigma} f_{1}^{p}(y)\right)^{\frac{1}{p}} \leq \frac{\theta_{2}}{\theta_{2}+1}\left({ }_{\varrho}^{y} \mathcal{K}_{r_{1}^{+}}^{\varsigma}\left(f_{1}+f_{2}\right)^{p}(y)\right)^{\frac{1}{p}}
$$

In contrast, as $m f_{2}(\eta) \leq f_{1}(\eta)$, it follows that

$$
\left(1+\frac{1}{\theta_{1}}\right)^{p} f_{2}^{p}(\eta) \leq\left(\frac{1}{\theta_{1}}\right)^{p}\left(f_{1}(\eta)+f_{2}(\eta)\right)^{p}
$$

Again, if we multiply both sides of (3.5) with $\frac{1}{\Gamma(\varsigma) \varsigma^{1-\tau-\varrho}}\left(\frac{y^{\tau+\varrho}-\eta^{\tau+\varrho}}{\tau+\varrho}\right)^{\tau-1}$ and then integrate the subsequent inequality with respect to $\eta$ from $r_{1}$ to $y$, we obtain

$$
\left(\begin{array}{l}
y \\
\varrho
\end{array} \mathcal{K}_{r_{1}^{+}}^{5} f_{2}^{p}(y)\right)^{\frac{1}{p}} \leq \frac{1}{\theta_{1}+1}\left({ }_{\varrho}^{y} \mathcal{K}_{r_{1}^{+}}^{5}\left(f_{1}+f_{2}\right)^{p}(y)\right)^{\frac{1}{p}}
$$

Thus adding (3.4) and (3.6) yields the desired inequality.

Inequality (3.1) is referred to as the reverse Minkowski inequality via generalized conformable fractional integrals.

Theorem 3.2 For $\varsigma>0, \varrho \in(0,1], \tau \in \mathcal{R}$ and $\varrho+\tau \neq 0$ with $p \geq 1$ let there be two positive functions $f_{1}, f_{2}$ on $[0, \infty)$ such that, for all $y>r_{1},{ }_{\varrho}^{\tau} \mathcal{K}_{r_{1}^{+}}^{5} f_{2}^{p}(y)<\infty$ and ${ }_{\varrho}^{y} \mathcal{K}_{r_{1}^{+}}^{5} f_{1}^{p}(y)<\infty$. If $0<\theta_{1} \leq \frac{f_{1}(\eta)}{f_{2}(\eta)} \leq \theta_{2}$ for $\theta_{1}, \theta_{2} \in \mathcal{R}^{+}$and for all $\eta \in\left[r_{1}, y\right]$, then

$$
\left({ }_{\varrho}^{y} \mathcal{K}_{r_{1}^{+}}^{\zeta} f_{1}^{p}(y)\right)^{\frac{2}{p}}+\left({ }_{\varrho}^{y} \mathcal{K}_{r_{1}^{+}}^{\varsigma} f_{2}^{p}(y)\right)^{\frac{2}{p}} \leq\left(\frac{\left(\theta_{1}+1\right)\left(\theta_{2}+1\right)}{\theta_{2}}-2\right)\left({ }_{\varrho}^{y} \mathcal{K}_{r_{1}^{+}}^{5} f_{1}^{p}(y)\right)^{\frac{1}{p}}\left({ }_{\varrho}^{y} \mathcal{K}_{r_{1}^{+}}^{5} f_{1}^{p}(y)\right)^{\frac{1}{p}}
$$

Proof The product of inequalities (3.4) and (3.6) yields

$$
\left(\frac{\left(\theta_{1}+1\right)\left(\theta_{2}+1\right)}{\theta_{2}}-2\right)\left({ }_{\varrho}^{y} \mathcal{K}_{r_{1}^{+}}^{\varsigma} f_{1}^{p}(y)\right)^{\frac{1}{p}}\left({ }_{\varrho}^{y} \mathcal{K}_{r_{1}^{+}}^{\varsigma} f_{2}^{p}(y)\right)^{\frac{1}{p}} \leq\left[\left({ }_{\varrho}^{y} \mathcal{K}_{r_{1}^{+}}^{\varsigma}\left(f_{1}+f_{2}\right)^{p}(y)\right)^{\frac{1}{p}}\right]^{2}
$$

Now, utilizing the Minkowski inequality to the right hand side of (3.8), one obtains

$$
\begin{aligned}
& {\left[\left({ }_{\varrho}^{y} \mathcal{K}_{r_{1}^{+}}^{5}\left(f_{1}+f_{2}\right)^{p}(y)\right)^{\frac{1}{p}}\right]^{2}} \\
& \left.\left.\quad \leq\left[{ }_{\varrho}^{y} \mathcal{K}_{r_{1}^{+}}^{5} f_{1}^{p}(y)\right)^{\frac{1}{p}}+{ }_{\varrho}^{y} \mathcal{K}_{r_{1}^{+}}^{5} f_{2}^{p}(y)\right)^{\frac{1}{p}}\right]^{2} \\
& \left.\left.\quad \leq\left({ }_{\varrho}^{y} \mathcal{K}_{r_{1}^{+}}^{5} f_{1}^{p}(y)\right)^{\frac{2}{p}}+\left({ }_{\varrho}^{y} \mathcal{K}_{r_{1}^{+}}^{5} f_{2}^{p}(y)\right)^{\frac{2}{p}}+2\left[{ }_{\varrho}^{y} \mathcal{K}_{r_{1}^{+}}^{5} f_{1}^{p}(y)\right)^{\frac{1}{p}}\right]\left[{ }_{\varrho}^{y} \mathcal{K}_{r_{1}^{+}}^{5} f_{2}^{p}(y)\right)^{\frac{1}{p}}\right] .
\end{aligned}
$$

Thus, from inequalities (3.8) and (3.9), we obtain the inequality (3.7). 


\section{Certain associated inequalities via generalized conformable fractional integral operators (GCFI)}

This section is dedicated to deriving certain associated variants regarding GCFI operator.

Theorem 4.1 For $\varsigma>0, \varrho \in(0,1], \tau \in \mathcal{R}, \varrho+\tau \neq 0$ with $p, q \geq 1$ and $\frac{1}{p}+\frac{1}{q}=1$, suppose that there are two positive functions $f_{1}, f_{2}$ on $[0, \infty)$ such that, for all $y>r_{1},{ }_{\varrho}^{\tau} \mathcal{K}_{r_{1}^{+}} f_{1}^{p}(y)<\infty$ and ${ }_{\varrho}^{y} \mathcal{K}_{r_{1}^{+}}^{5} f_{2}^{p}(y)<\infty$. If $0<\theta_{1} \leq \frac{f_{1}(\eta)}{f_{2}(\eta)} \leq \theta_{2}$ for $\theta_{1}, \theta_{2} \in \mathcal{R}^{+}$and for all $\eta \in\left[r_{1}, y\right]$, then

$$
\left({ }_{\varrho}^{y} \mathcal{K}_{r_{1}^{+}}^{\varsigma} f_{1}^{p}(y)\right)^{\frac{1}{p}}\left({ }_{\varrho}^{y} \mathcal{K}_{r_{1}^{+}}^{\varsigma} f_{2}^{q}(y)\right)^{\frac{1}{q}} \leq\left(\frac{\theta_{2}}{\theta_{1}}\right)^{\frac{1}{p q}}\left({ }_{\varrho}^{y} \mathcal{K}_{r_{1}^{+}}^{\varsigma} f_{1}^{\frac{1}{p}}(y) f_{2}^{\frac{1}{q}}(y)\right)
$$

Proof Under the given suppositions $\frac{f_{1}(\eta)}{f_{2}(\eta)} \leq \theta_{2}, r_{1} \leq \eta \leq y$, therefore we have

$$
f_{2}^{\frac{1}{q}}(\eta) \geq \theta_{2}^{\frac{-1}{q}} f_{1}^{\frac{1}{q}}(\eta)
$$

Taking the product of both sides of $(4.2)$ by $f_{1}^{\frac{1}{p}}(\eta)$, it follows that

$$
f_{1}^{\frac{1}{p}}(\eta) f_{2}^{\frac{1}{q}}(\eta) \geq \theta_{2}^{\frac{-1}{q}} f_{1}(\eta)
$$

If we multiply both sides of (4.3) with $\frac{1}{\Gamma(\varsigma) \varsigma^{1-\tau-\varrho}}\left(\frac{y^{\tau+\varrho}-\eta^{\tau+\varrho}}{\tau+\varrho}\right)^{\tau-1}$ and then integrate the subsequent inequality with respect to $\eta$ from $r_{1}$ to $y$, we obtain

$$
\begin{aligned}
& \frac{\theta_{2}^{\frac{-1}{q}}}{\Gamma(\varsigma)} \int_{r_{1}}^{y}\left(\frac{y^{\tau+\varrho}-\eta^{\tau+\varrho}}{\tau+\varrho}\right)^{\tau-1} \frac{f_{1}(\eta) d \eta}{\varsigma^{1-\tau-\varrho}} \\
& \leq \frac{1}{\Gamma(\varsigma)} \int_{r_{1}}^{y}\left(\frac{y^{\tau+\varrho}-\eta^{\tau+\varrho}}{\tau+\varrho}\right)^{\tau-1} \frac{f_{1}^{\frac{1}{p}}(\eta) f_{2}^{\frac{1}{q}}(\eta) d \eta}{\varsigma^{1-\tau-\varrho}} .
\end{aligned}
$$

Consequently, we have

$$
\theta_{2}^{-\frac{1}{p q}}\left({ }_{\varrho}^{y} \mathcal{K}_{r_{1}^{+}}^{\varsigma} f_{1}^{p}(y)\right)^{\frac{1}{p}} \leq\left({ }_{\varrho}^{y} \mathcal{K}_{r_{1}^{+}}^{\zeta} f_{1}^{\frac{1}{p}}(y) f_{2}^{\frac{1}{q}}(y)\right)^{\frac{1}{p}}
$$

In contrast, as $\theta_{1} f_{2}(\eta) \leq f_{1}(\eta)$, we have

$$
\theta_{1}^{\frac{1}{\bar{p}}} f_{2}^{\frac{1}{\bar{p}}}(\eta) \leq f_{1}^{\frac{1}{\bar{p}}}(\eta)
$$

Again, if we multiply both sides of (4.6) by $f_{2}^{\frac{1}{q}}(\eta)$ and invoke the relation $\frac{1}{p}+\frac{1}{q}=1$, it yields

$$
\theta_{1}^{\frac{1}{p}} f_{2}(\eta) \leq f_{1}^{\frac{1}{p}}(\eta) f_{2}^{\frac{1}{q}}(\eta)
$$

If we multiply both sides of (4.7) with $\frac{1}{\Gamma(\varsigma) \varsigma^{1-\tau-\varrho}}\left(\frac{y^{\tau+\varrho}-\eta^{\tau+\varrho}}{\tau+\varrho}\right)^{\tau-1}$ and then integrate the subsequent inequality with respect to $\eta$ from $r_{1}$ to $y$, we obtain

$$
\theta_{1}^{\frac{1}{p q}}\left({ }_{\varrho}^{y} \mathcal{K}_{r_{1}^{+}}^{\varsigma} f_{2}(y)\right)^{\frac{1}{q}} \leq\left(\begin{array}{l}
y \\
\varrho
\end{array} \mathcal{K}_{r_{1}^{+}}^{5} f_{1}^{\frac{1}{p}}(y) f_{2}^{\frac{1}{q}}(y)\right)^{\frac{1}{q}}
$$

Multiplying (4.5) and (4.8), the required inequality (4.1) can be concluded. 
Theorem 4.2 For $\varsigma>0, \varrho \in(0,1], \tau \in \mathcal{R}, \varrho+\tau \neq 0$ with $p, q \geq 1$ and $\frac{1}{p}+\frac{1}{q}=1$. Suppose that there are two positive functions $f_{1}, f_{2}$ on $[0, \infty)$ such that, for all $y>r_{1},{ }_{e}^{\tau} \mathcal{K}_{r_{1}^{+}}^{5} f_{1}^{p}(y)<\infty$ and ${ }_{\varrho}^{y} \mathcal{K}_{r_{1}^{+}}^{s} f_{2}^{p}(y)<\infty$. If $0<\theta_{1} \leq \frac{f_{1}(\eta)}{f_{2}(\eta)} \leq \theta_{2}$ for $\theta_{1}, \theta_{2} \in \mathcal{R}^{+}$and for all $\eta \in\left[r_{1}, y\right]$, then

$$
\left.\left({ }_{\varrho}^{y} \mathcal{K}_{r_{1}^{+}}^{\varsigma} f_{1}(y) f_{2}(y)\right) \leq \frac{2^{p-1} \theta_{2}^{p}}{p\left(\theta_{2}+1\right)^{p}}{ }_{\varrho}^{y} \mathcal{K}_{r_{1}^{+}}^{\varsigma}\left(f_{1}^{p}+f_{2}^{p}\right)(y)\right)+\frac{2^{q-1}}{p\left(\theta_{1}+1\right)^{p}}\left({ }_{\varrho}^{y} \mathcal{K}_{r_{1}^{+}}^{\varsigma}\left(f_{1}^{q}+f_{2}^{q}\right)(y)\right) .
$$

Proof By the given assumption $\frac{f_{1}(\eta)}{f_{2}(\eta)}<\theta_{2}$, we have

$$
\left(\theta_{2}+1\right)^{p} f_{1}^{p}(\eta) \leq \theta_{2}^{p}\left(f_{1}+f_{2}\right)^{p}(\eta)
$$

If we multiply both sides of (4.10) with $\frac{1}{\Gamma(\varsigma) \varsigma^{1-\tau-\varrho}}\left(\frac{y^{\tau+Q}-\eta^{\tau+\varrho}}{\tau+\varrho}\right)^{\tau-1}$ and then integrate the subsequent inequality with respect to $\eta$ from $r_{1}$ to $y$, we obtain

$$
\begin{aligned}
& \frac{\left(\theta_{2}+1\right)^{p}}{\Gamma(\varsigma)} \int_{r_{1}}^{y}\left(\frac{y^{\tau+\varrho}-\eta^{\tau+\varrho}}{\tau+\varrho}\right)^{\tau-1} \frac{f_{1}^{p}(\eta) d \eta}{\varsigma^{1-\tau-\varrho}} \\
& \leq \frac{\theta_{2}^{p}}{\Gamma(\varsigma)} \int_{r_{1}}^{y}\left(\frac{y^{\tau+\varrho}-\eta^{\tau+\varrho}}{\tau+\varrho}\right)^{\tau-1} \frac{\left(f_{1}+f_{2}\right)^{p}(\eta) d \eta}{\varsigma^{1-\tau-\varrho}} .
\end{aligned}
$$

It follows that

$$
\left({ }_{\varrho}^{y} \mathcal{K}_{r_{1}^{+}}^{\zeta} f_{1}^{p}(y)\right) \leq \frac{\theta_{2}^{p}}{\left(\theta_{2}+1\right)^{p}}\left({ }_{\varrho}^{y} \mathcal{K}_{r_{1}^{+}}^{\varsigma}\left(f_{1}+f_{2}\right)^{p}(y)\right)
$$

In contrast, using $0<\theta_{1} \leq \frac{f_{1}(\eta)}{f_{2}(\eta)}, r_{1}<\eta<y$, we have

$$
\left(\theta_{1}+1\right)^{q} f_{2}^{q}(\eta) \leq\left(f_{1}+f_{2}\right)^{q}(\eta)
$$

Again, if we multiply both sides of (4.13) with $\frac{1}{\Gamma(\varsigma) \varsigma^{1-\tau-\varrho}}\left(\frac{y^{\tau+\varrho}-\eta^{\tau+\varrho}}{\tau+\varrho}\right)^{\tau-1}$ and then integrate the subsequent inequality with respect to $\eta$ from $r_{1}$ to $y$, we obtain

$$
\left({ }_{\varrho}^{y} \mathcal{K}_{r_{1}^{+}}^{5} f_{2}^{q}(y)\right) \leq \frac{1}{\left(\theta_{1}+1\right)^{q}}\left({ }_{\varrho}^{y} \mathcal{K}_{r_{1}^{+}}^{5}\left(f_{1}+f_{2}\right)^{q}(y)\right)
$$

Now, taking into account Young's inequality,

$$
f_{1}(\eta) f_{2}(\eta) \leq \frac{f_{1}^{p}(\eta)}{p}+\frac{f_{2}^{q}(\eta)}{q}
$$

Now, if we multiply both sides of (4.15) with $\frac{1}{\Gamma(\varsigma) \varsigma^{1-\tau-\varrho}}\left(\frac{y^{\tau+\varrho}-\eta^{\tau+\varrho}}{\tau+\varrho}\right)^{\tau-1}$ and then integrate the subsequent inequality with respect to $\eta$ from $r_{1}$ to $y$, we obtain

$$
\left({ }_{\varrho}^{y} \mathcal{K}_{r_{1}^{+}}^{\zeta}\left(f_{1} f_{2}\right)(y)\right) \leq \frac{1}{p}\left({ }_{\varrho}^{y} \mathcal{K}_{r_{1}^{+}}^{\zeta} f_{1}^{p}(y)\right)+\frac{1}{q}\left({ }_{\varrho}^{y} \mathcal{K}_{r_{1}}^{\zeta} f_{2}^{q}(y)\right) .
$$


With the aid of (4.12) and (4.14) with (4.16), one obtains

$$
\begin{aligned}
& \left({ }_{\varrho}^{y} \mathcal{K}_{r_{1}^{+}}^{\zeta}\left(f_{1} f_{2}\right)(y)\right) \\
& \leq \frac{1}{p}\left({ }_{\varrho}^{y} \mathcal{K}_{r_{1}^{+}}^{\zeta} f_{1}^{p}(y)\right)+\frac{1}{q}\left({ }_{\varrho}^{y} \mathcal{K}_{r_{1}^{+}}^{\zeta} f_{2}^{q}(y)\right) \\
& \leq \frac{\theta_{2}^{p}}{p\left(\theta_{2}+1\right)^{p}}\left(\begin{array}{l}
y \\
\varrho
\end{array} \mathcal{K}_{r_{1}^{+}}^{5}\left(f_{1}+f_{2}\right)^{p}(y)\right)+\frac{1}{q\left(\theta_{1}+1\right)^{q}}\left({ }_{\varrho}^{y} \mathcal{K}_{r_{1}^{+}}^{5}\left(f_{1}+f_{2}\right)^{q}(y)\right) .
\end{aligned}
$$

Using the inequality $(\mu+v)^{s} \leq 2^{s-1}\left(\mu^{s}+v^{s}\right), s>1, \mu, v>0$, one can obtain

$$
\left({ }_{\varrho}^{y} \mathcal{K}_{r_{1}^{+}}^{\varsigma}\left(f_{1}+f_{2}\right)^{p}(y)\right) \leq 2^{p-1}\left({ }_{\varrho}^{y} \mathcal{K}_{r_{1}^{+}}^{\varsigma}\left(f_{1}^{p}+f_{2}^{p}\right)(y)\right)
$$

and

$$
\left({ }_{\varrho}^{y} \mathcal{K}_{r_{1}^{+}}^{\varsigma}\left(f_{1}+f_{2}\right)^{q}(y)\right) \leq 2^{q-1}\left({ }_{\varrho}^{y} \mathcal{K}_{r_{1}^{+}}^{\varsigma}\left(f_{1}^{q}+f_{2}^{q}\right)(y)\right)
$$

Hence, the proof of (4.9) can be concluded from (4.17), (4.18), and (4.19) collectively.

Theorem 4.3 For $\varsigma>0, \varrho \in(0,1], \tau \in \mathcal{R}, \varrho+\tau \neq 0$ with $p \geq 1$ and let there be two positive functions $f_{1}, f_{2}$ on $[0, \infty)$ such that, for all $y>r_{1},{ }_{\varrho}^{\tau} \mathcal{K}_{r_{1}^{+}}^{5} f_{1}^{p}(y)<\infty$ and ${ }_{\varrho}^{y} \mathcal{K}_{r_{1}^{+}}^{\mathcal{S}_{2}} f_{2}^{p}(y)<\infty$. If $0<\lambda<\theta_{1} \leq \frac{f_{1}(\eta)}{f_{2}(\eta)} \leq \theta_{2}$ for $\theta_{1}, \theta_{2} \in \mathcal{R}^{+}$and for all $\eta \in\left[r_{1}, y\right]$, then

$$
\begin{aligned}
& \frac{\theta_{2}+1}{\theta_{2}-\lambda}\left({ }_{\varrho}^{y} \mathcal{K}_{r_{1}^{+}}^{5}\left(f_{1}(y)-\lambda f_{2}(y)\right)\right) \leq\left({ }_{\varrho}^{y} \mathcal{K}_{r_{1}^{+}}^{5}\left(f_{1}\right)^{p}(y)\right)^{\frac{1}{p}}+\left({ }_{\varrho}^{y} \mathcal{K}_{r_{1}^{+}}^{5}\left(f_{2}\right)^{p}(y)\right)^{\frac{1}{p}} \\
& \leq \frac{\theta_{1}+1}{\theta_{1}-\lambda}\left({ }_{\varrho}^{y} \mathcal{K}_{r_{1}^{+}}^{\varsigma}\left(f_{1}(y)-\lambda f_{2}(y)\right)\right)^{\frac{1}{p}} .
\end{aligned}
$$

Proof Under the given supposition $0<\lambda<\theta_{1} \leq \frac{f_{1}^{p}(\eta)}{f_{2}^{p}(\eta)} \leq \theta_{2}$, we have

$$
\begin{aligned}
\theta_{1} \lambda \leq \theta_{2} \lambda & \Rightarrow \quad \theta_{1} \lambda+\theta_{1} \leq \theta_{1} \lambda+\theta_{2} \leq \theta_{2} \lambda+\theta_{2} \\
& \Rightarrow \quad\left(\theta_{2}+1\right)\left(\theta_{1}-\lambda\right) \leq\left(\theta_{1}+1\right)\left(\theta_{2}-\lambda\right) .
\end{aligned}
$$

It follows that

$$
\frac{\theta_{2}+1}{\theta_{2}-\lambda} \leq \frac{\theta_{1}+1}{\theta_{1}-\lambda}
$$

Also, we have

$$
\theta_{1}-\lambda \leq \frac{f_{1}(\eta)-\lambda f_{2}(\eta)}{f_{2}(\eta)} \leq \theta_{2}-\lambda,
$$

implying

$$
\frac{\left(f_{1}(\eta)-\lambda f_{2}(\eta)\right)^{p}}{\left(\theta_{2}-\lambda\right)^{p}} \leq f_{2}^{p}(\eta) \leq \frac{\left(f_{1}(\eta)-\lambda f_{2}(\eta)\right)^{p}}{\left(\theta_{1}-\lambda\right)^{p}}
$$


Furthermore, we have

$$
\frac{1}{\theta_{2}} \leq \frac{f_{2}(\eta)}{f_{1}(\eta)} \leq \frac{1}{\theta_{1}} \Rightarrow \frac{\theta_{1}-\lambda}{\lambda \theta_{1}} \leq \frac{f_{1}(\eta)-\lambda f_{2}(\eta)}{\lambda f_{1}(\eta)} \leq \frac{\theta_{2}-\lambda}{\theta_{2} \lambda} .
$$

It follows that

$$
\left(\frac{\theta_{2}}{\theta_{2}-\lambda}\right)^{p}\left(f_{1}(\eta)-\lambda f_{2}(\eta)\right)^{p} \leq f_{1}^{p}(\eta) \leq\left(\frac{\theta_{1}}{\theta_{1}-\lambda}\right)^{p}\left(f_{1}(\eta)-\lambda f_{2}(\eta)\right)^{p} .
$$

If we multiply both sides of (4.22) with $\frac{1}{\Gamma(\varsigma) \varsigma^{1-\tau-\varrho}}\left(\frac{y^{\tau+Q}-\eta^{\tau+\varrho}}{\tau+\varrho}\right)^{\tau-1}$ and then integrate the subsequent inequality with respect to $\eta$ from $r_{1}$ to $y$, we obtain

$$
\begin{aligned}
& \frac{1}{\left(\theta_{2}-\lambda\right)^{p} \Gamma(\varsigma)} \int_{r_{1}}^{y}\left(\frac{y^{\tau+\varrho}-\eta^{\tau+\varrho}}{\tau+\varrho}\right)^{\tau-1} \frac{\left(f_{1}(\eta)-\lambda f_{2}(\eta)\right)^{p} d \eta}{\varsigma^{1-\tau-\varrho}} \\
& \leq \frac{1}{\Gamma(\varsigma)} \int_{r_{1}}^{y}\left(\frac{y^{\tau+\varrho}-\eta^{\tau+\varrho}}{\tau+\varrho}\right)^{\tau-1} \frac{\left(f_{2}^{p}(\eta)\right) d \eta}{\varsigma^{1-\tau-\varrho}} \\
& \leq \frac{1}{\left(\theta_{1}-\lambda\right)^{p} \Gamma(\varsigma)} \int_{r_{1}}^{y}\left(\frac{y^{\tau+\varrho}-\eta^{\tau+\varrho}}{\tau+\varrho}\right)^{\tau-1} \frac{\left(f_{1}(\eta)-\lambda f_{2}(\eta)\right)^{p} d \eta}{\varsigma^{1-\tau-\varrho}} .
\end{aligned}
$$

Accordingly, it can be written as

$$
\begin{aligned}
\frac{1}{\theta_{2}-\lambda}\left(\varrho_{\varrho}^{y} \mathcal{K}_{r_{1}^{+}}^{\zeta}\left(f_{1}(y)-\lambda f_{2}(y)\right)^{p}\right)^{\frac{1}{p}} & \leq\left({ }_{\varrho}^{y} \mathcal{K}_{r_{1}^{+}}^{\zeta}\left(f_{2}\right)^{p}(y)\right)^{\frac{1}{p}} \\
& \leq \frac{1}{\theta_{1}-\lambda}\left({ }_{\varrho}^{y} \mathcal{K}_{r_{1}^{+}}^{\zeta}\left(f_{1}(y)-\lambda f_{2}(y)\right)^{p}\right)^{\frac{1}{p}} .
\end{aligned}
$$

Adopting the same technique with (4.22), one obtains

$$
\begin{aligned}
\frac{\theta_{2}}{\theta_{2}-\lambda}\left({ }_{\varrho}^{y} \mathcal{K}_{r_{1}^{+}}^{\zeta}\left(f_{1}(y)-\lambda f_{2}(y)\right)^{p}\right)^{\frac{1}{p}} & \leq\left({ }_{\varrho}^{y} \mathcal{K}_{r_{1}^{+}}^{\zeta} f_{1}^{p}(y)\right)^{\frac{1}{p}} \\
& \leq \frac{\theta_{1}}{\theta_{1}-\lambda}\left(\begin{array}{l}
y \\
\varrho
\end{array} \mathcal{K}_{r_{1}^{+}}^{\zeta}\left(f_{1}(y)-\lambda f_{2}(y)\right)^{p}\right)^{\frac{1}{p}} .
\end{aligned}
$$

Hence, by adding inequalities (4.23) and (4.24), we attain the inequality (4.20).

Theorem 4.4 For $\varsigma>0, \varrho \in(0,1], \tau \in \mathcal{R}, \varrho+\tau \neq 0$ with $p \geq 1$ and let there are two positive functions $f_{1}, f_{2}$ on $[0, \infty)$ such that, for all $y>r_{1},{ }_{\varrho}^{\tau} \mathcal{K}_{r_{1}^{+}}^{\zeta} f_{1}^{p}(y)<\infty$ and ${ }_{\varrho}^{y} \mathcal{K}_{r_{1}^{+}}^{5} f_{2}^{p}(y)<\infty$. If $0<\hbar \leq f_{1}(\eta) \leq \mathcal{H}$ and $0<\mathfrak{m} \leq f_{2}(\eta) \leq \mathcal{M}$ for $\theta_{1}, \theta_{2} \in \mathcal{R}^{+}$and for all $\eta \in\left[r_{1}, y\right]$, then

$$
\left({ }_{\varrho}^{y} \mathcal{K}_{r_{1}}^{y} f_{1}^{p}(\eta)\right)^{\frac{1}{p}}+\left({ }_{\varrho}^{y} \mathcal{K}_{r_{1}}^{y} f_{2}^{p}(\eta)\right)^{\frac{1}{p}} \leq \frac{\mathcal{H}(\hbar+\mathcal{M})+\mathcal{M}(\mathcal{H}+\mathfrak{m})}{(\mathfrak{m}+\mathcal{H})(\hbar+\mathcal{M})}\left(\varrho_{\varrho}^{y} \mathcal{K}_{r_{1}}^{y}\left(f_{1}+f_{2}\right)^{p}(\eta)\right)^{\frac{1}{p}} .
$$

Proof Under the given suppositions, observe that

$$
\frac{1}{\mathcal{M}} \leq \frac{1}{f_{2}(\eta)} \leq \frac{1}{\mathfrak{m}}
$$

Conducting the product between (4.26) and $0 \leq \hbar \leq f_{1}(\eta) \leq \mathcal{H}$, we have

$$
\frac{\hbar}{\mathcal{M}} \leq \frac{f_{1}(\eta)}{f_{2}(\eta)} \leq \frac{\mathcal{H}}{\mathfrak{m}} .
$$


From (4.27), we obtain

$$
f_{2}^{p}(\eta) \leq\left(\frac{\mathcal{M}}{\hbar+\mathcal{M}}\right)^{p}\left(f_{1}(\eta)+f_{2}(\eta)\right)^{p}
$$

and

$$
f_{1}^{p}(\eta) \leq\left(\frac{\mathcal{H}}{\mathfrak{m}+\mathcal{H}}\right)^{p}\left(f_{1}(\eta)+f_{2}(\eta)\right)^{p}
$$

If we multiply both sides of (4.28) with $\frac{1}{\Gamma(\varsigma) \varsigma^{1-\tau-\varrho}}\left(\frac{y^{\tau+\varrho}-\eta^{\tau+\varrho}}{\tau+\varrho}\right)^{\tau-1}$ and then integrate the subsequent inequality with respect to $\eta$ from $r_{1}$ to $y$, we obtain

$$
\begin{aligned}
& \frac{1}{\Gamma(\varsigma)} \int_{r_{1}}^{y}\left(\frac{y^{\tau+\varrho}-\eta^{\tau+\varrho}}{\tau+\varrho}\right)^{\tau-1} \frac{f_{2}^{p}(\eta) d \eta}{\varsigma^{1-\tau-\varrho}} \\
& \leq \frac{\mathcal{M}^{p}}{(\hbar+\mathcal{M})^{p} \Gamma(\varsigma)} \int_{r_{1}}^{y}\left(\frac{y^{\tau+\varrho}-\eta^{\tau+\varrho}}{\tau+\varrho}\right)^{\tau-1} \frac{\left(f_{1}(y)+f_{2}(y)\right)^{p}(\eta) d \eta}{\varsigma^{1-\tau-\varrho}} .
\end{aligned}
$$

Accordingly,

$$
\left({ }_{\varrho}^{y} \mathcal{K}_{r_{1}}^{y} f_{2}^{p}(y)\right)^{\frac{1}{p}} \leq \frac{\mathcal{M}}{\hbar+\mathcal{M}}\left({ }_{\varrho}^{y} \mathcal{K}_{r_{1}}^{y}\left(f_{1}+f_{2}\right)^{p}(y)\right)^{\frac{1}{p}}
$$

Adopting the same technique as (4.29), one obtains

$$
\left({ }_{\varrho}^{y} \mathcal{K}_{r_{1}}^{y} f_{1}^{p}(y)\right)^{\frac{1}{p}} \leq \frac{\mathcal{H}}{\mathfrak{m}+\mathcal{H}}\left({ }_{\varrho}^{y} \mathcal{K}_{r_{1}}^{y}\left(f_{1}+f_{2}\right)^{p}(y)\right)^{\frac{1}{p}}
$$

Hence, by adding (4.31) and (4.32), we obtain the inequality (4.25).

Theorem 4.5 For $\varsigma>0, \varrho \in(0,1], \tau \in \mathcal{R}, \varrho+\tau \neq 0$ with $p \geq 1$ let there be two positive functions $f_{1}, f_{2}$ on $[0, \infty)$ such that, for all $y>r_{1},{ }_{\varrho}^{\tau} \mathcal{K}_{r_{1}^{+}}^{5} f_{1}^{p}(y)<\infty$ and ${ }_{\varrho}^{y} \mathcal{K}_{r_{1}^{+}}^{5} f_{1}^{p}(y)<\infty$. If $0<\hbar \leq f_{1}(\eta) \leq \mathcal{H}$ and $0<\mathfrak{m} \leq f_{2}(\eta) \leq \mathcal{M}$ for $\theta_{1}, \theta_{2} \in \mathcal{R}^{+}$for all $\eta \in\left[r_{1}, y\right]$, then

$$
\begin{aligned}
\frac{1}{\theta_{2}}\left({ }_{\varrho}^{y} \mathcal{K}_{r_{1}}^{y} f_{1}(y) f_{2}(y)\right) & \leq \frac{1}{\left(\theta_{1}+1\right)\left(\theta_{2}+1\right)}\left({ }_{\varrho}^{y} \mathcal{K}_{r_{1}}^{y}\left(f_{1}(y)+f_{2}(y)\right)\right)^{2} \\
& \leq \frac{1}{\theta_{1}}\left({ }_{\varrho}^{y} \mathcal{K}_{r_{1}}^{y} f_{1}(y) f_{2}(y)\right) .
\end{aligned}
$$

Proof Under the given suppositions, $0<\theta_{1} \leq \frac{f_{1}(\eta)}{f_{2}(\eta)} \leq \theta_{2}$, it follows that

$$
f_{2}(\eta)\left(\theta_{1}+1\right) \leq f_{1}(\eta)+f_{2}(\eta) \leq f_{2}(\eta)\left(\theta_{2}+1\right) .
$$

Additionally, we have $\frac{1}{\theta_{2}} \leq \frac{f_{2}(\eta)}{f_{1}(\eta)} \leq \frac{1}{\theta_{1}}$, which yields

$$
\left(\frac{\theta_{2}+1}{\theta_{2}}\right) f_{1}(\eta) \leq f_{1}(\eta)+f_{2}(\eta) \leq\left(\frac{\theta_{1}+1}{\theta_{1}}\right) f_{1}(\eta)
$$

The product of (4.34) and (4.35) gives

$$
\frac{f_{1}(\eta) f_{2}(\eta)}{\theta_{2}} \leq \frac{\left(f_{1}(\eta)+f_{2}(\eta)\right)^{2}}{\left(\theta_{1}+1\right)\left(\theta_{2}+1\right)} \leq \frac{f_{1}(\eta) f_{2}(\eta)}{\theta_{1}} .
$$


Now, if we multiply both sides of (4.36) with $\frac{1}{\Gamma(5) \varsigma^{1-\tau-\varrho}}\left(\frac{y^{\tau+\varrho}-\eta^{\tau+\varrho}}{\tau+\varrho}\right)^{\tau-1}$ and then integrate the subsequent inequality with respect to $\eta$ from $r_{1}$ to $y$, we obtain

$$
\begin{aligned}
& \frac{1}{\theta_{2} \Gamma(\varsigma)} \int_{r_{1}}^{y}\left(\frac{y^{\tau+\varrho}-\eta^{\tau+\varrho}}{\tau+\varrho}\right)^{\tau-1} \frac{f_{1}(\eta) f_{2}(\eta) d \eta}{\varsigma^{1-\tau-\varrho}} \\
& \leq \frac{1}{\Gamma(\varsigma)\left(\left(\theta_{1}+1\right)\left(\theta_{2}+1\right)\right)} \int_{r_{1}}^{y}\left(\frac{y^{\tau+\varrho}-\eta^{\tau+\varrho}}{\tau+\varrho}\right)^{\tau-1} \frac{\left(f_{1}(\eta)+f_{2}(\eta)\right)^{2} d \eta}{\varsigma^{1-\tau-\varrho}} \\
& \leq \frac{1}{\theta_{1} \Gamma(\varsigma)} \int_{r_{1}}^{y}\left(\frac{y^{\tau+\varrho}-\eta^{\tau+\varrho}}{\tau+\varrho}\right)^{\tau-1} \frac{f_{1}(\eta) f_{2}(\eta) d \eta}{\varsigma^{1-\tau-\varrho}}
\end{aligned}
$$

One observes that

$$
\frac{1}{\theta_{2}}\left({ }_{e}^{y} \mathcal{K}_{r_{1}}^{y} f_{1}(y) f_{2}(y)\right) \leq \frac{1}{\left(\theta_{1}+1\right)\left(\theta_{2}+1\right)}\left({ }_{\varrho}^{y} \mathcal{K}_{r_{1}}^{y}\left(f_{1}(y)+f_{2}(y)\right)\right)^{2} \leq \frac{1}{\theta_{1}}\left({ }_{\varrho}^{y} \mathcal{K}_{r_{1}}^{y} f_{1}(y) f_{2}(y)\right),
$$

which is the desired result.

Theorem 4.6 For $\varsigma>0, \varrho \in(0,1], \tau \in \mathcal{R}, \varrho+\tau \neq 0$ with $p \geq 1$ and let there are two positive functions $f_{1}, f_{2}$ on $[0, \infty)$ such that, for all $y>r_{1},{ }_{\varrho}^{\tau} \mathcal{K}_{r_{1}^{+}}^{5} f_{1}^{p}(y)<\infty$ and ${ }_{\varrho}^{y} \mathcal{K}_{r_{1}^{+}}^{5} f_{2}^{p}(y)<\infty$. If $0<\hbar \leq f_{1}(\eta) \leq \mathcal{H}$ and $0<\theta_{1} \leq f_{2}(\eta) \leq \theta_{2}$ for $\theta_{1}, \theta_{2} \in \mathcal{R}^{+}$and for all $\eta \in\left[r_{1}, y\right]$, then

$$
\left({ }_{\varrho}^{y} \mathcal{K}_{r_{1}^{+}}^{\zeta} f_{1}^{p}(y)\right)^{\frac{1}{p}}+\left({ }_{\varrho}^{y} \mathcal{K}_{r_{1}^{+}}^{\varsigma} f_{2}^{p}(y)\right)^{\frac{1}{p}} \leq 2\left({ }_{\varrho}^{y} \mathcal{K}_{r_{1}^{+}}^{\zeta} \mathcal{U}^{p}\left(f_{1}(y), f_{2}(y)\right)\right)^{\frac{1}{p}},
$$

where $\mathcal{U}\left(f_{1}(\eta), f_{2}(\eta)\right)=\max \left\{\theta_{2}\left[\left(1+\frac{\theta_{2}}{\theta_{1}}\right) f_{1}(y)-\theta_{2} f_{2}(y)\right], \frac{\left(\theta_{1}+\theta_{2}\right) f_{2}(y)-f_{1}(y)}{\theta_{1}}\right\}$.

Proof Under the given suppositions $0<\theta_{1} \leq \frac{f_{1}(\eta)}{f_{2}(\eta)} \leq \theta_{2}, r_{1} \leq \eta \leq y$, we have

$$
0<\theta_{1} \leq \theta_{2}+\theta_{1}-\frac{f_{1}(\eta)}{f_{2}(\eta)}
$$

and

$$
\theta_{2}+\theta_{1}-\frac{f_{1}(\eta)}{f_{2}(\eta)} \leq \theta_{2}
$$

From (4.38) and (4.39), one obtains

$$
f_{2}(\eta)<\frac{\left(\theta_{2}+\theta_{1}\right) f_{2}(\eta)-f_{1}(\eta)}{\theta_{1}} \leq \mathcal{U}\left(f_{1}(\eta), f_{2}(\eta)\right)
$$

where $\mathcal{U}\left(f_{1}(\eta), f_{2}(\eta)\right)=\max \left\{\theta_{2}\left[\left(1+\frac{\theta_{2}}{\theta_{1}}\right) f_{1}(y)-\theta_{2} f_{2}(y)\right], \frac{\left(\theta_{1}+\theta_{2}\right) f_{2}(y)-f_{1}(y)}{\theta_{1}}\right\}$. Also, from the given supposition $0<\frac{1}{\theta_{2}} \leq \frac{f_{2}(\eta)}{f_{1}(\eta)} \leq \frac{1}{\theta_{1}}$, one has

$$
\frac{1}{\theta_{2}} \leq \frac{1}{\theta_{2}}+\frac{1}{\theta_{1}}-\frac{f_{2}(\eta)}{f_{1}(\eta)}
$$

and

$$
\frac{1}{\theta_{2}}+\frac{1}{\theta_{1}}-\frac{f_{2}(\eta)}{f_{1}(\eta)} \leq \frac{1}{\theta_{1}}
$$


From (4.41) and (4.42), we get

$$
\frac{1}{\theta_{2}} \leq \frac{\left(\frac{1}{\theta_{1}}+\frac{1}{\theta_{2}}\right) f_{1}(\eta)-f_{2}(\eta)}{f_{1}(\eta)} \leq \frac{1}{\theta_{1}}
$$

implying

$$
\begin{aligned}
f_{1}(\eta) & \leq \theta_{2}\left(\frac{1}{\theta_{1}}+\frac{1}{\theta_{2}}\right) f_{1}(\eta)-\theta_{2} f_{2}(\eta) \\
& =\frac{\theta_{2}\left(\theta_{1}+\theta_{2}\right) f_{1}(\eta)-\theta_{2}^{2} \theta_{1} f_{2}(\eta)}{\theta_{1} \theta_{2}} \\
& =\left(\frac{\theta_{2}}{\theta_{1}}+1\right) f_{1}(\eta)-\theta_{2} f_{2}(\eta) \\
& \leq \theta_{2}\left[\left(\frac{\theta_{2}}{\theta_{1}}+1\right) f_{1}(\eta)-\theta_{2} f_{2}(\eta)\right] \\
& \leq \mathcal{U}\left(f_{1}(\eta), f_{2}(\eta)\right) .
\end{aligned}
$$

From (4.40) and (4.44), we have

$$
f_{1}^{p}(\eta) \leq \mathcal{U}^{p}\left(f_{1}(\eta), f_{2}(\eta)\right)
$$

and

$$
f_{2}^{p}(\eta) \leq \mathcal{U}^{p}\left(f_{1}(\eta), f_{2}(\eta)\right) .
$$

If we multiply both sides of (4.45) with $\frac{1}{\Gamma(\varsigma) \varsigma^{1-\tau-\varrho}}\left(\frac{y^{\tau+\varrho}-\eta^{\tau+\varrho}}{\tau+\varrho}\right)^{\tau-1}$ and then integrate the subsequent inequality with respect to $\eta$ from $r_{1}$ to $y$, we obtain

$$
\begin{aligned}
& \frac{1}{\Gamma(\varsigma)} \int_{r_{1}}^{y}\left(\frac{y^{\tau+\varrho}-\eta^{\tau+\varrho}}{\tau+\varrho}\right)^{\tau-1} \frac{f_{1}^{p}(\eta) d \eta}{\varsigma^{1-\tau-\varrho}} \\
& \leq \frac{1}{\Gamma(\varsigma)} \int_{r_{1}}^{y}\left(\frac{y^{\tau+\varrho}-\eta^{\tau+\varrho}}{\tau+\varrho}\right)^{\tau-1} \frac{\mathcal{U}^{p}\left(f_{1}(\eta), f_{2}(\eta)\right) d \eta}{\varsigma^{1-\tau-\varrho}}
\end{aligned}
$$

Accordingly,

$$
\left({ }_{\varrho}^{y} \mathcal{K}_{r_{1}^{+}}^{\varsigma} f_{1}^{p}(y)\right)^{\frac{1}{p}} \leq\left({ }_{\varrho}^{y} \mathcal{K}_{r_{1}^{+}}^{\varsigma} \mathcal{U}^{p}\left(f_{1}(y), f_{2}(y)\right)\right)^{\frac{1}{p}}
$$

Adopting the same technique for (4.46), we have

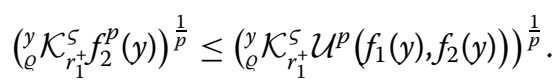

Hence, by adding (4.48) and (4.49), we obtain the inequality (4.37).

\section{Concluding remarks}

This paper begins with a compact evaluation of fractional integrals in the sense of Riemann-Liouville and Riemann-Liouville type conformable fractional integral operators in addition to a new fractional integral operator according to Khan et al. [14]. We 
generalize the reverse Minkowski inequalities via generalized conformable fractional integrals; specifically, the inequality concerning fractional integrals in the Riemann-Liouville sense is given [44]. The associated significant variants regarding generalized conformable fractional integrals are demonstrated. Numerous variants can be established for the application of several defined fractional integral operators. One of the well-known inequalities is the Chebyshev inequality lately derived in [38]. Finally, this concept can be extended in the form of a $\mathcal{K}$ analogue for deriving similar types of results and these are also helpful for establishing the refinements of several existing results in the literature.

\section{Acknowledgements \\ The authors would like to express their sincere thanks to the editor and the anonymous reviewers for their helpful comments and suggestions.}

\section{Funding}

The author T. Abdeljawad would like to thank Prince Sultan University for funding this work through research group Nonlinear Analysis Methods in Applied Mathematics (NAMAM) group number RG-DES-2017-01-17.

\section{Availability of data and materials}

Not applicable.

\section{Competing interests}

The authors declare that they have no competing interests.

\section{Authors' contributions}

All authors contributed equally to the writing of this paper. All authors read and approved the final manuscript.

\section{Author details}

${ }^{1}$ Department of Mathematics, Government College University, Faisalabad, Pakistan. ${ }^{2}$ Department of Mathematics, Faculty of Science and Letters, Agrı Ibrahim Cecen University, 04100 Agri, Turkey. ${ }^{3}$ Department of Mathematics, College of Arts and Sciences, Prince Sattam bin Abdulaziz University, Wadi Aldawaser, Saudi Arabia. ${ }^{4}$ Department of Mathematics and General Sciences, Prince Sultan University, 66833 Riyadh, Saudi Arabia. ${ }^{5}$ Department of Medical Research, China Medical University, 40402 Taichung, Taiwan. ${ }^{6}$ Department of Computer Science and Information Engineering, Asia University, 40402 Taichung, Taiwan. ${ }^{7}$ Department of Mathematics, Shaheed Benazir Bhutto University, 18000 Sheringal, Upper Dir, Pakistan.

\section{Publisher's Note}

Springer Nature remains neutral with regard to jurisdictional claims in published maps and institutional affiliations.

Received: 5 May 2020 Accepted: 17 June 2020 Published online: 29 June 2020

\section{References}

1. Abdeljawad, T.: On conformable fractional calculus. J. Comput. Appl. Math. 279, 57-66 (2015)

2. Khalil, R., Al Horani, M., Yousef, A., Sababheh, M.: A new definition of fractional derivative. J. Comput. Appl. Math. 264, 65-70 (2014)

3. Khan, H., Gómez-Aguilar, J.F., Alkhazzan, A., Khan, A.: A fractional order HIV-TB coinfection model with nonsingular Mittag-Leffler law. Math. Methods Appl. Sci. 43(6), 3786-3806 (2020)

4. Khan, H., Khan, A., Jarad, F., Anwar, S.: Existence and data dependence theorems for solutions of an ABC-fractional order impulsive system. Chaos Solitons Fractals 18, 109477 (2019)

5. Khan, A., Khan, H., Gómez-Aguilar, J.F., Abdeljawad, T.: Existence and Hyers-Ulam stability for a nonlinear singular fractional differential equations with Mittag-Leffler kernel. Chaos Solitons Fractals 127(1), 422-427 (2019)

6. Yavuz, M., Özdemir, N.: Comparing the new fractional derivative operators involving exponential and Mittag-Leffler kernel. Discrete Contin. Dyn. Syst., Ser. S 13(3), 995 (2020)

7. Yavuz, M., Özdemir, N.: European vanilla option pricing model of fractional order without singular kernel. Fractal Fract. 2(1), 3 (2018)

8. Miller, K.S., Ross, B.: An Introduction to the Fractional Calculus and Fractional Differential Equations. Wiley, New York (1993)

9. Munkhammar, J.D.: Riemann-Liouville fractional derivatives and the Taylor-Riemann Series, Examensarbete i matematik, 10 poang Handledare och examinator: Andreas Strombergsson Juni (2004)

10. Podlubny, I.: Fractional Differential Equations. Academic Press, San Diego (1999)

11. Kilbas, S.G., Marichev, A.A.: Fractional Integrals and Derivatives, Theory and Applications. Gordon \& Breach, Yverdon (1993)

12. Jarad, F., Ugurlu, U., Abdeljawad, T., Baleanu, D.: On a new class of fractional operators. Adv. Differ. Equ. 2017, 247 (2017)

13. Anderson, D.R., Ulness, D.J.: Newly defined conformable derivatives. Adv. Dyn. Syst. Appl. 58, 109-137 (2015)

14. Khan, T.U., Khan, M.A.: Generalized conformable fractional integral operators. J. Comput. Appl. Math. 346, 378-389 (2019) 
15. Kilbas, A.A., Srivastava, H.M., Trujillo, J.J.: Theory and Applications of Fractional Differential Equations. North-Holland Mathematical Studies, North-Holland, Amsterdam (2006)

16. Abdeljawad, T., Jarad, F., Alzabut, J.: Fractional proportional differences with memory. Eur. Phys. J. Spec. Top. 226 3333-3354 (2017)

17. Khan, H., Jarad, F., Abdeljawad, T., Khan, A.: A singular ABC-fractional differential equation with $p$-Laplacian operator. Chaos Solitons Fractals 129(1), 56-61 (2019)

18. Khan, A., Gómez-Aguilar, J.F., Khan, T.S., Khan, H.: Stability analysis and numerical solutions of fractional order HIV/AIDS model. Chaos Solitons Fractals 122, 119-128 (2019)

19. Yavuz, M.: Characterizations of two different fractional operators without singular kernel. Math. Model. Nat. Phenom. 14(3), 302 (2019)

20. Yavuz, M.: Novel solution methods for initial boundary value problems of fractional order with conformable differentiation. Int. J. Optim. Control Theor. Appl. 8, 1, 1-7 (2017)

21. Yavuz, M., Bonyah, E.: New approaches to the fractional dynamics of schistosomiasis disease model. Physica A 525, 373-393 (2019)

22. Jarad, F., Abdeljawad, T., Alzabut, J.: Generalized fractional derivatives generated by a class of local proportional derivatives. Eur. Phys. J. Spec. Top. 226, 3457-3471 (2017)

23. Qian, W.-M., Yang, Y.-Y., Zhang, H.-W., Chu, Y.-M.: Optimal two-parameter geometric and arithmetic mean bounds for the Shandor-Yang mean. J. Inequal. Appl. 2019, Article ID 287 (2019)

24. Wang, M.-K., Chu, Y.-M., Zhang, W.: Precise estimates for the solution of Ramanujan's generalized modular equation. Ramanujan J. 49(3), 653-668 (2019)

25. Adil Khan, M., Zaheer Ullah, S., Chu, Y.-M.: The concept of co-ordinate strongly convex functions and related inequalities. Rev. R. Acad. Cienc. Exactas Fís. Nat., Ser. A Mat. 113(3), 2235-2251 (2019)

26. Zaheer Ullah, S., Adil Khan, M., Khan, Z.A., Chu, Y.-M.: Integral majorization type inequalities for the functions in the sense of strong convexity. J. Funct. Spaces 2019, Article ID 9487823 (2019)

27. Bainov, D.D. Simeonov, P.S. Integral Inequalities and Applications, vol. 57. Springer, Berlin (2013)

28. Bandle, C., Gilanyi, A., Losonczi, A., Pales, Z., Plum, M.: Inequalities and Applications: Conference on Inequalities and Applications, Noszvaj (Hungary), September 2007, vol. 157. Springer, Berlin (2008)

29. Agarwal, R.P., Zbekler, A.O.: Lyapunov type inequalities for mixed nonlinear Riemann-Liouville fractional differential equations with a forcing term. J. Comput. Appl. Math. 314, 69-78 (2017)

30. Rashid, S., Jarad, F., Kalsoom, H., Chu, Y.-M.: On Pólya-Szegö and Čebyšev type inequalities via generalized $\mathcal{K}$-fractional integrals. Adv. Differ. Equ. 2020, Article ID 125 (2020). https://doi.org/10.1186/s13662

31. Rashid, S., Noor, M.A., Noor, K.I., Chu, Y.-M.: Ostrowski type inequalities in the sense of generalized $\mathcal{K}$-fractiona integral operator for exponentially convex functions. AIMS Math. 5(3), 2629-2645 (2020) https://doi.org/10.3934/math.2020171

32. Rafeeq, S., Kalsoom, H., Hussain, S., Rashid, S., Chu, Y.-M.: Delay dynamic double integral inequalities on time scales with applications. Adv. Differ. Equ. 2020, Article ID 40 (2020)

33. Latif, M.A. Rashid, S., Dragomir, S.S., Chu, Y.M. Hermite-Hadamard type inequalities for co-ordinated convex and quasi-convex functions and their applications. J. Inequal. Appl. 2019, Article ID 317 (2019). https://doi.org/10.1186/s13660-019-2272-7

34. Wang, M.-K., Chu, Y.-M., Zhang, W.: Monotonicity and inequalities involving zero-balanced hypergeometric function. Math. Inequal. Appl. 22(2), 601C617 (2019)

35. Abbas Baloch, I., Chu, Y.-M.: Petrovic-type inequalities for harmonic $h$-convex functions. J. Funct. Spaces 2020, Article ID 3075390, 7 pages (2020)

36. Khan, M.A., Khurshid, Y., Du, T.-S., Chu, Y.-M.: Generalization of Hermite-Hadamard type inequalities via conformable fractional integrals. J. Funct. Spaces 2018, Article ID 5357463 (2018)

37. Rashid, S., Jarad, F., Chu, Y.-M.: A note on reverse Minkowski inequality via generalized proportional fractional integral operator with respect to another function. Math. Probl. Eng. 2020, Article ID 7630260 (2020). https://doi.org/10.1155/2020/7630260

38. Nisar, S.K., Rahman, G., Mehrez, K.: Čebyšev type inequalities via generalized fractional conformable integrals. J. Inequal. Appl. 2019, 245 (2019)

39. Dahmani, Z: New inequalities in fractional integrals. Int. J. Nonlinear Sci. 9(4), 493-497 (2010)

40. Rashid, S., Hammouch, Z., Kalsoom, H., Ashraf, R., Chu, Y.-M.: New investigation on the generalized $\mathcal{K}$-fractional integral operators. Front. Phys. 8, 25 (2020). https://doi.org/10.3389/fphy.2020.00025

41. Rashid, S., Ashraf, R., Noor, M.A., Noor, K.I., Chu, Y.-M.: New weighted generalizations for differentiable exponentially convex mapping with application. AIMS Math. 5(4), 3525-3546 (2020). https://doi.org/10.3934/math.2020229

42. Liu, W.J., Ngo, Q.A., Huy, V.N.: Several interesting integral inequalities. J. Math. Inequal. 3, 201-212 (2009)

43. Bougoffa, L.: On Minkowski and Hardy integral inequalities. J. Inequal. Pure Appl. Math. 7, Article ID 60 (2006)

44. Dahmani, Z:: On Minkowski and Hermite-Hadamard integral inequalities via fractional integral. Ann. Funct. Anal. 1, $51-58(2010)$

45. Rashid, S., Noor, M.A., Noor, K.I., Safdar, F., Chu, Y.-M.: Hermite-Hadamard type inequalities for the class of convex functions on time scale. Mathematics 7, 956 (2019). https://doi.org/10.3390/math7100956

46. Rashid, S., Jarad, F., Noor, M.A., Kalsoom, H., Chu, Y.-M.: Inequalities by means of generalized proportional fractional integral operators with respect to another function. Mathematics 7, 1225 (2020). https://doi.org/10.3390/math7121225

47. Rashid, S., Latif, M.A., Hammouch, Z., Chu, Y.-M.: Fractional integral inequalities for strongly $h$-preinvex functions for a kth order differentiable functions. Symmetry 11, 1448 (2019). https://doi.org/10.3390/sym11121448

48. Khan, H., Abdeljawad, T., Tunç, C., Alkhazzan, A., Khan, A.: Minkowski's inequality for the AB-fractional integral operator. J. Inequal. Appl. 2019, 96 (2019)

49. Mohammed, P.O., Abdeljawad, T.: Modification of certain fractional integral inequalities for convex functions. Adv. Differ. Equ. 2020, 69 (2020)

50. Zhou, S.-S., Rashid, S., Dragomir, S.S., Latif, M.A., Akdemir, A.O., Liu, J.-B.: Some new inequalities involving $\mathcal{K}$-fractional integral for certain classes of functions and their applications. J. Funct. Spaces 2020, Article ID 5285147 (2020). https://doi.org/10.1155/2020/5285147 
51. Rashid, S., Jarad, F., Noor, M.A., Noor, K.I., Baleanu, D., Liu, J.-B.: On Gruss inequalities within generalized $\mathcal{K}$-fractional integrals. Adv. Differ. Equ. 2020, 203 (2020). https://doi.org/10.1186/s13662-020-02644-7

52. Rashid, S., AbdelJawad, T., Jarad, F., Noor, M.A.: Some estimates for generalized Riemann-Liouville fractional integrals of exponentially convex functions and their applications. Mathematics 7(9), 807 (2019). https://doi.org/10.3390/math7090807

53. Mubeen, S., Habib, S., Naeem, M.N.: The Minkowski inequality involving generalized k-fractional conformable integral, J. Inequal. Appl. 2019, 81 (2019). https://doi.org/10.1186/s13660-019-2040-8

54. Set, E., Tomar, M., Sarikaya, M.Z.: On generalized Gruss type inequalities for K-fractional integrals. Appl. Math. Comput. 269, 29-34 (2015)

55. Wang, G.-D., Zhang, X.-H., Chu, Y.-M.: A power mean inequality for the Grötzsch ring function. Math. Inequal. Appl. 14(4), 833-837 (2011)

56. Qiu, Y.-F., Wang, M.-K., Chu, Y.-M., Wang, G.-D.: Two sharp inequalities for Lehmer mean, identric mean and logarithmic mean. J. Math. Inequal. 5(3), 301-306 (2011)

57. Chu, Y.-M., Long, B.-Y.: Sharp inequalities between means. Math. Inequal. Appl. 14(3), 647-655 (2011)

58. Mohammed, P.O.: Hermite-Hadamard inequalities for Riemann-Liouville fractional integrals of a convex function with respect to a monotone function. Math. Methods Appl. Sci. (2019, in press). https://doi.org/10.1002/mma.5784

59. Mohammed, P.O., Brevik, I.: A new version of the Hermite-Hadamard inequality for Riemann-Liouville fractional integrals. Symmetry 12, 610 (2020). https://doi.org/10.3390/sym12040610

60. Set, E., Ozdemir, M., Dragomir, S.: On the Hermite-Hadamard inequality and other integral inequalities involving two functions. J. Inequal. Appl. 2010, 148102 (2010)

\section{Submit your manuscript to a SpringerOpen ${ }^{\circ}$ journal and benefit from:}

- Convenient online submission

- Rigorous peer review

- Open access: articles freely available online

- High visibility within the field

- Retaining the copyright to your article

Submit your next manuscript at $\boldsymbol{\nabla}$ springeropen.com 